\title{
Las Redes de Nueva Generación: ¿un nuevo modelo para las telecomunicaciones en España?*
}

\author{
Juan José Ganuza \\ Universitat Pompeu Fabra (UPF) \\ Karla Perca \\ Toulouse School of Economics (TSE) \\ María Fernanda Viecens \\ Fundación de Estudios de Economía Aplicada (FEDEA)
}

\begin{abstract}
Resumen
Las Redes de Acceso de Nueva Generación (Next Generation Access Networks, NGANs) basadas en fibra óptica implican una verdadera revolución tecnológica para el sector de las telecomunicaciones. Reemplazar el cobre por fibra permite mejorar drásticamente las prestaciones de las redes actuales, alcanzando velocidades de acceso de más de 100 Mbps e incrementando el número y la calidad de los servicios que se prestan. Además, el despliegue de estas redes presenta nuevos desafíos para la industria que frente a factores que estaban ausentes con la red de cobre, se enfrenta ahora a situaciones que pueden modificar por completo su estructura. Estos desafios permiten la entrada de nuevos actores en el sector y obligan a los operadores tradicionales a replantear sus modelos tradicionales de negocio.

Este artículo ofrece una introducción técnica a las NGANs, discute por qué la llegada de las NGANs está cambiando la estructura de la industria de telecomunicaciones y señala cuáles son los principales elementos diferenciadores con respecto a la industria basada en la red de cobre. A continuación se revisa la realidad española en relación al despliegue de estas redes y en perspectiva con el contexto internacional. El artículo muestra que el despliegue de las NGANs en España es limitado. Esta situación es preocupante, no sólo porque no se está aprovechando el potencial impacto económico de las NGANs, sino porque además la renovación de las redes sería una oportunidad para aumentar la competencia dentro del sector.
\end{abstract}

Palabras clave: Redes de Acceso de Nueva Generación (NGANs), telecomunicaciones, regulación.

Clasificación JEL: K23, L50, L96.

\begin{abstract}
The Next Generation Access Networks (NGANs) based on optical fiber mean a revolution for the telecommunications sector. Replacing copper with fiber dramatically improves the performance of existing networks, allowing for speeds of over $100 \mathrm{Mbps}$ and increasing the number and quality of services provided. In addition, the deployment of these networks presents new challenges for the industry that, due to the presence of new elements that were absent in the industry based on the copper network, is faced with situations that can completely change its structure. These challenges permit the entry of new agents in the sector, and incumbents are forced to rethink their traditional business models. This article provides a technical introduction to the NGANs, discusses why the

* Los autores agradecen los comentarios de los asistentes al Workshop en Economía de las Telecomunicaciones (CMT-Fedea-UPF), Barcelona, CMT, marzo de 2011. Juan-José Ganuza agradece la hospitalidad de Fedea, así como la financiación del Ministerio Español de Ciencia e Innovación bajo el proyecto SEC2003-08080-C02-01. M. Fernanda Viecens agradece la financiación del Ministerio Español de Ciencia e Innovación bajo el proyecto ECO2009-07530.
\end{abstract}


arrival of the NGANs is changing the structure of the telecommunications industry and identifies the key differences with respect to the industry based on the copper network. Then, we review the Spanish situation in relation to the deployment of these networks and in perspective with the international context. This paper shows that the deployment of NGANs in Spain is limited. This is worrying not only because Spain is not exploiting the potential economic impact of the NGANs, but also because the renewal of the networks would be an opportunity to increase competition within the sector.

Keywords: Next Generation Access Networks (NGANs), telecommunications, regulation.

JEL classification: K23, L50, L96.

\section{Introducción}

Las redes de cobre han sido la base de las telecomunicaciones en los últimos 100 años. En la actualidad asistimos a una revolución tecnológica en el sector con la sustitución de dichas redes por las denominadas redes de acceso de nueva generación (Next Generation Access Networks, NGANs) basadas en fibra óptica. Reemplazar el cobre por fibra óptica permite mejorar drásticamente las prestaciones de las redes actuales, alcanzando velocidades de acceso de más de $100 \mathrm{Mbps}$ y esto podría tener un efecto muy importante sobre la economía en su conjunto, fundamentalmente por cuatro razones.

1. Nuevas aplicaciones. El incremento del ancho de banda permitirá la aparición de nuevas aplicaciones en la industria del ocio y de los contenidos audiovisuales. Las posibilidades que se abren para todas las aplicaciones basadas en Internet (P2P, juegos online, etc.) y nuevos servicios de multimedia como la HDTV bajo demanda, son enormes ${ }^{1}$. Para el ámbito público, son importantes además las aplicaciones de las NGANs en el sector educativo (e-learning) y en el sanitario (e-health, especialmente telemedicina). Las aplicaciones de elearning permiten superar las barreras geográficas ya que estas redes hacen posible impartir clases en videoconferencia a una audiencia ubicada en distintas áreas geográficas, con buena calidad de comunicación y con posibilidad de interacción en tiempo real. En cuanto a la telemedicina, las redes de nueva generación abren la posibilidad de realizar consultas médicas, diagnósticos y monitoreo de los pacientes a distancia. Estas aplicaciones pueden ser especialmente relevantes para personas con movilidad reducida o que viven en zonas alejadas de los centros con tecnología de punta ${ }^{2}$.

${ }^{1}$ La capacidad de simultaneidad ilustra las ventajas de las NGN y el amplio abanico de futuras aplicaciones que abren. Por ejemplo, hacer uso de la telefonía IP requiere, aproximadamente, de $0,5 \mathrm{Mbps}$ de subida y bajada, y ver en un solo televisor un canal de HDTV necesita 8 Mbps de bajada y 0,5 Mbps de subida. Esto quiere decir que sólo para ver un canal de televisión en alta definición y, al mismo tiempo, realizar una llamada telefónica mediante telefonía IP se necesitan 8,5 Mbps de bajada y 1 Mbps de subida. El uso simultáneo de otras aplicaciones añadiría Mbps a esta suma (GAPTEL, 2008).

2 Algunas de estas aplicaciones ya están siendo puestas en práctica. En España se han realizado algunas pruebas de consulta y diagnóstico a distancia. Uno de estos casos son las pruebas del sistema HealthPresence de Cisco, diseñado para enviar datos en audio, vídeo e información médica a un médi- 
2. Impacto en la productividad y capacidad de innovación. Las NGANs fomentarán el teletrabajo, el cloud-computing y las teleconferencias, reduciendo las barreras geográficas y los costes de transporte ${ }^{3}$. Además, estas redes podrían contribuir a la aparición de nuevos sectores industriales ${ }^{4}$.

3. Aumento de la actividad económica y creación de empleo. Una gran cantidad de artículos e informes documentan la existencia de un efecto positivo de la inversión en infraestructura de telecomunicaciones en el crecimiento económico. Véase Holt y Jamison (2009) y Koutroumpis (2009) para una revisión de la literatura ${ }^{5}$. Hay dos aspectos que hacen comparativamente más atractiva las inversiones en NGAN que en otro tipo de infraestructuras: i) Extender estas redes en zonas sin cobertura o con déficit de cobertura podría tener efectos marginales más fuertes en la oferta y productividad que simplemente realizar mejoras a las redes de servicios públicos ya existentes (OCDE, 2009) ${ }^{6}$. ii) Casi el 70 por 100 de la inversión en NGANs es atribuible a la mano de obra, por lo tanto el impacto positivo en la creación de puestos de trabajo puede ser muy importante ${ }^{7}$.

co localizado en una ubicación distinta a la del paciente. Además, los resultados de las pruebas realizadas por un centro médico pueden ser enviados vía Internet a cualquier especialista disponible para hacer el diagnóstico. Este tipo de tecnologías permite ahorrar costes y tiempo. En particular, los tiempos se pueden reducir de cuatro o cinco días a 30 minutos. En Francia, el desarrollo de $e$-health es considerado una prioridad nacional (véase http://esante.gouv.fr). Para estimaciones de los beneficios potenciales de implementación de programas de telemedicina en Estados Unidos y Australia véase MITCHELL y PEZZULLO (2010). Por ejemplo, ellos estiman que los beneficios para Australia de la aplicación a gran escala de la telemedicina podrían ser cercanos a los 1,5-2,9 miles de millones de euros al año.

${ }^{3}$ FORNEFEL et al., (2008) estima que en Europa la incorporación de tecnologías de información basadas en banda ancha en el sector de manufacturas podría llevar a incrementos en productividad de 5 por 100 . Asimismo, la posibilidad de proveer servicios por Internet podría llevar a incrementos en la productividad del sector servicios de 10 por 100 . Del mismo modo, en cuanto al sector público, se pueden esperar ahorros en los sistemas de salud de 20,1 mil millones de euros (EU-27) por el uso de sistemas de salud electrónica basados en banda ancha.

4 SIMES et al., (2010) presentan los resultados de una encuesta llevada a cabo en Australia sobre el impacto que las empresas esperan de la construcción de la red de fibra. Los principales resultados incluyen: casi el 50 por 100 de los encuestados espera poder ofrecer diferentes tipos de productos y servicios; el 57 por 100 de los encuestados espera cambios en la forma de comunicarse con proveedores y clientes; además el 20 por 100 de los encuestados cree que la red nacional de fibra permitirá diferentes modelos de empleo.

5 KOUTROUMPIS (2009) presenta además nuevos resultados específicos sobre el impacto económico de la infraestructura de banda ancha. En particular, encuentra que el 3,91 por 100 del crecimiento en España durante el período 2002-2007 puede ser atribuido a la infraestructura en banda ancha. Nótese que en otros países como Italia y Portugal ese porcentaje es cercano al 16 por 100, o inclusive más alto (alrededor del 19 por 100 en Holanda y Suiza).

${ }^{6}$ De cualquier manera, a la hora de decidir sobre un proyecto de despliegue siempre debería llevarse a cabo un análisis coste-beneficio.

7 KATZ y SUTER (2009) estiman que, bajo un escenario optimista, la inversión en banda ancha de 6.390 millones de dólares del paquete estímulo de inversión de los Estados Unidos generará 273.000 empleos para el período 2009-2012. Por otra parte, KATZ et al., (2009) cuantifican el impacto macroeconómico de la inversión en tecnología de banda ancha en el empleo y la producción de la economía de Alemania que implicará la «Estrategia de Banda Ancha Nacional» anunciada por el gobierno. Los 
4. Cambios en la estructura del sector de las telecomunicaciones. El objetivo central de este artículo, es analizar el impacto que las NGANs tendrán en la industria. Nuestra principal conclusión es que las NGANs representan un «nuevo mundo» que cambiará completamente la cadena de valor y al que los operadores tradiciones de telecomunicaciones tendrán que saber adaptarse para mantener una posición de liderazgo en el mercado. En particular, se identifican y analizan cuatro elementos que sustentan esta afirmación: 1) la descentralización y viabilidad de redes pequeñas, 2) la separación estructural, 3) la intervención pública y 4) el impacto de los mercados online.

A continuación desarrollamos una descripción técnica de lo que es una red de nueva generación y presentamos también su estructura y los agentes implicados. En la sección 3 discutimos los cuatro factores fundamentales que a nuestro juicio están generando un nuevo modelo de industria. En la sección 4 evaluamos la situación en España. Revisamos los despliegues y proyectos que existen en este momento y presentamos un análisis del país en perspectiva internacional. La sección 5 presenta las conclusiones.

\section{Descripción de una red de acceso de nueva generación}

\subsection{Descripción técnica}

La constante evolución tecnológica en el ámbito de las comunicaciones y la información ha permitido ofrecer incrementos en las velocidades de conexión a Internet, con el consecuente desarrollo de contenidos y aplicaciones que utilizan al mismo tiempo cada vez más ancho de banda. Las tecnologías xDSL han aprovechado al máximo la capacidad de las redes tradicionales de cobre para ofrecer velocidades de hasta $30 \mathrm{Mbps}$, al mismo tiempo que las redes de cable han ido mejorando para incrementar también sus velocidades. Sin embargo, las redes de acceso de nueva generación representan un salto más allá de la evolución de una tecnología sustentada sobre las redes tradicionales.

No existe una definición única de las redes de acceso de nueva generación. La Comisión Europea las define como «redes de acceso cableadas que consisten total o parcialmente en elementos ópticos y son capaces de prestar servicios de acceso de banda ancha con características mejoradas en comparación con los servicios prestados a través de las redes de cobre existentes». ${ }^{8}$

autores sostienen que el impacto del plan para un período de 10 años será la creación de 968.000 nuevos puestos de trabajo y un incremento del Producto Interno Bruto en 170,9 mil millones de euros. Véase también HOLT y JAMISON (2009) para una discusión sobre el impacto de la banda ancha en la creación de empleo.

8 COMISIÓN EUROPEA (2009a), esta definición sólo es aplicable a las redes físicas, e ignora a las redes inalámbricas. 
Las redes de acceso de nueva generación acercan la fibra óptica a los usuarios finales, lo que permite velocidades muy superiores a las que se pueden alcanzar mediante el tradicional ADSL. Según la distancia entre la fibra y el usuario final se pueden distinguir varios tipos de despliegue, denominados de manera general como FTTx (véase el Gráfico 1). Al respecto, se identifican:

- Fibre-to-the-Home (FTTH): En este tipo de accesos la fibra óptica llega hasta la vivienda del usuario, lo que permite alcanzar velocidades superiores a los 100 Mbps.

- Fibre-to-the-Building (FTTB): En este caso la fibra óptica llega hasta el exterior del edificio y luego se utiliza cobre para llegar hasta el domicilio de cada usuario final. Las velocidades que se pueden alcanzar con este tipo de acceso pueden llegar a los 100 Mbps.

- Fibre-to-the-Node (FTTN) o Fiber to the Cabinet (FTTC): En estos accesos la fibra llega hasta un nodo cercano al usuario final y a partir de allí la red continúa mediante cobre. Debido a que el último tramo es de cobre, con estos accesos se pueden alcanzar velocidades bastante inferiores a las correspondientes a FTTH o FTTB.

Al mismo tiempo, cuando se habla de redes de acceso de nueva generación no se puede dejar de lado a las redes de cable HFC (Híbrido de Fibra y Coaxial). El desarrollo del estándar DOCSIS 3.0 ha hecho posible que estas redes sean capaces de ofrecer velocidades de $100 \mathrm{Mbps}$. Esto permite que los operadores de cable compitan prácticamente en igualdad de condiciones con las redes de FTTx en las zonas donde ambos tipos de infraestructuras están presentes. Asimismo, el proceso de actualización a DOCSIS 3.0 tiene un coste muy bajo si se lo compara con el que deben afrontar las empresas que actualmente cuentan con la infraestructura de ADSL. Esto se debe a que las operadoras de cable no necesitan desplegar una nueva red, mientras que en las redes de ADSL es necesario reemplazar los tramos de cobre por fibra óptica. 


\section{GRÁFICO 1 \\ TIPOS DE DESPLIEGUE DE FTTx}

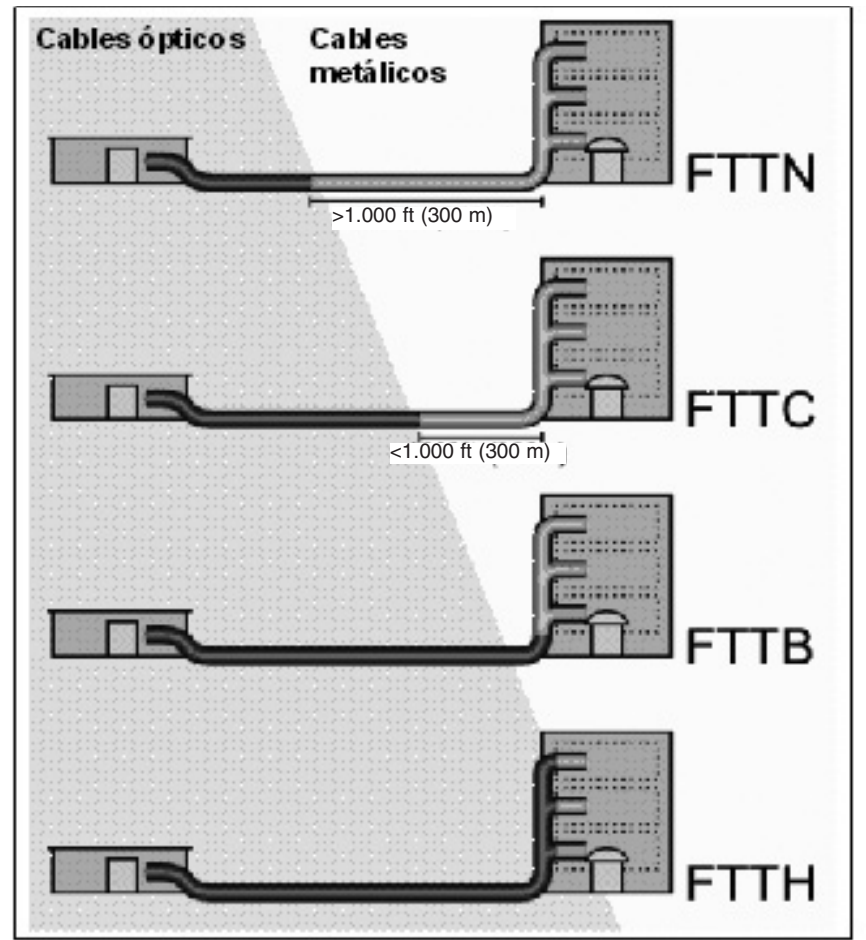

FUENTE: WIKIMEDIA COMMONS.

\subsection{Principales agentes de la red}

La industria está comprendida básicamente por los tres niveles que se muestran en el Gráfico 2: la infraestructura pasiva, la infraestructura activa y los servicios a los usuarios finales. 
GRÁFICO 2 AGENTES Y NIVELES DE LA RED

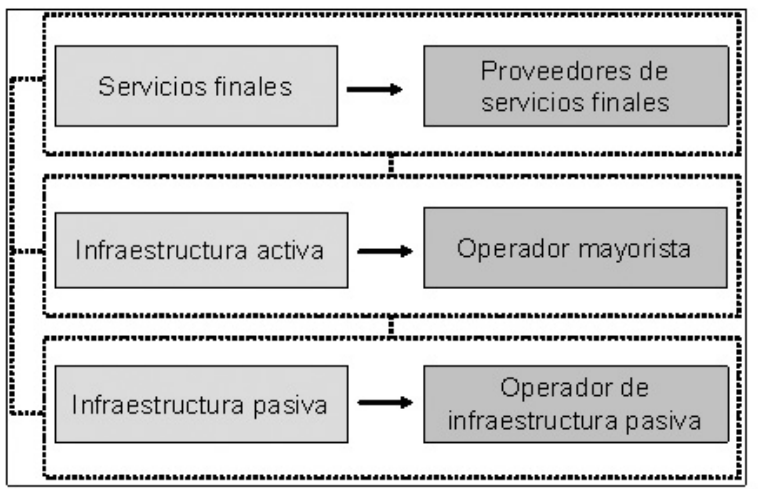

El diseño de esta estructura como así también la relación que se establezca entre los operadores de cada nivel desempeñará un papel fundamental en el resultado de mercado. Asimismo, los efectos sobre el bienestar de los consumidores vendrán determinados por el nivel de integración entre los diferentes segmentos. Analizamos a continuación las funciones y agentes presentes en cada segmento.

\subsubsection{Infraestructura pasiva}

La infraestructura pasiva está comprendida por los cables de fibra óptica, los conductos y la obra civil en general. Es el segmento que requiere los mayores esfuerzos de inversión. Sin embargo, la tecnología a este nivel es relativamente estándar por lo que no proporciona claras posibilidades de diferenciación ni potencial de innovación. La empresa que invierte para diseñar y construir esta red es generalmente la misma que luego se hará cargo de su mantenimiento y operación. Se espera que con excepción de algunas ciudades grandes y densamente pobladas esta infraestructura será un monopolio. Por consiguiente, un consumidor que pretenda tener acceso a servicios que requieren un gran ancho de banda estará, por lo general, «atrapado» en una única red de acceso.

\subsubsection{Infraestructura activa}

La infraestructura activa comprende el equipamiento técnico que permite ofrecer los servicios mayoristas a los proveedores de servicios finales. El operador a cargo controla la calidad de los servicios para los consumidores y la gestión del tráfico dentro de la red. 


\subsubsection{Servicios finales}

Este nivel incluye la amplia variedad de servicios que los consumidores pueden adquirir de los operadores de telecomunicaciones tales como servicios de voz, televisión, Internet y todo tipo de contenido digital. Los operadores pagan un precio de acceso para usar la red y contratan directamente con los usuarios finales para la provisión de los servicios. Se espera que éste sea el segmento con mayor progreso tecnológico y potencial de innovación y es en este nivel donde los operadores deberán buscar elementos de diferenciación.

\section{Consecuencias de las NGNs para la estructura del sector}

A continuación analizamos ciertos factores que implican importantes novedades para el sector, en el sentido de que han estado prácticamente ausentes en la industria basada en la red de cobre, y que por consiguiente con su aparición se traducen en serias transformaciones para la industria.

\subsection{Redes descentralizadas y viabilidad de redes pequeñas}

Si bien existen importantes economías de escala y densidad a nivel local en el despliegue de la fibra óptica, a diferencia de lo que sucedía con la red de cobre heredada por el incumbente ya no es indispensable contar con una red centralizada. Ésta es una de las consecuencias principales de la tecnología IP. Las redes tradicionales de cobre se sustentaban sobre una red nacional que fue en su momento un monopolio natural. Los cambios tecnológicos (principalmente el desarrollo de las tecnologías de la información) permitieron liberalizar las telecomunicaciones, y compatibilizar la inversión de múltiples operadores en conexiones de larga y media distancia con la red del operador incumbente, que no obstante mantenía el control sobre el bucle del abonado. La inversión en el bucle del abonado no era factible, no solamente por la duplicidad del coste fijo, sino también por la necesidad de coordinación con el resto de la red ${ }^{9}$. Las NGANs implican reemplazar el bucle de cobre, y además no requieren dicha coordinación. Por lo tanto, redes de menor tamaño y cobertura local pueden también ser eficientes, y de hecho, estamos asistiendo a la aparición de numerosos proyectos de alcance limitado (véase la siguiente sección para casos en España). Esta característica de las NGANs tiene la ventaja de abrir el mercado a un número mayor de agentes, pero el surgimiento de gran cantidad de proyectos aislados puede no estar exento de problemas. La carencia de una política

\footnotetext{
${ }^{9}$ Esta afirmación es real para el cobre aunque no necesariamente lo es también para el caso del cable.
} 
a nivel nacional que actúe al menos como elemento unificador de las diversas actuaciones podría desencadenar ineficiencias principalmente vinculadas a la compatibilidad tecnológica.

\subsection{Desintegración vertical y separación estructural}

La definición de NGN utilizada por la Unión Internacional de Telecomunicaciones reconoce la separación entre servicios y transporte ${ }^{10}$. Esta característica que pone de manifiesto una diferencia fundamental en relación a las redes de cobre heredadas por las empresas incumbentes reduce las economías de alcance de proveer conjuntamente la infraestructura y los servicios a los consumidores finales. Esto nos lleva a pensar que las sinergias tecnológicas existentes entre los diferentes segmentos no son lo suficientemente fuertes como para justificar redes completamente integradas. Al respecto, Doyle (2008) sostiene que el mayor énfasis en la diversidad de servicios plantea la importancia de concentrar el esfuerzo en el segmento minorista y sospecha que las empresas podrían encontrar hasta beneficioso especializarse y separar sus actividades minoristas de sus actividades de la red al por mayor. Esto sugiere que la gestión de este nivel será la más importante a medida que la naturaleza de los productos finales se vuelva más sofisticada. Cave (2006) propone una clasificación de los diferentes tipos de separación vertical que se pueden plantear. Establece seis modalidades entre, y además de, la separación contable y la completa separación de la propiedad (separación estructural) ${ }^{11}$.

Al respecto no existe en la literatura un consenso generalizado sobre la conveniencia de implementar la separación funcional o estructural en el sector de las telecomunicaciones, y existen fuertes argumentos tanto a favor como en contra de estas medidas. Cadman (2010) ofrece una revisión de la literatura acerca de los posibles efectos de la integración vertical y de los diferentes tipos de separación. En general, los estudios coinciden en que cuando la empresa que opera la red es dominante tiene fuertes incentivos para perjudicar a los rivales que prestan servicios a través de su red. Éste es el principal argumento a favor de la separación. En el caso particular de

\footnotetext{
${ }^{10}$ La definición de la UIT es la siguiente: A Next Generation Network (NGN) is a packet-based network able to provide services including Telecommunication Services and able to make use of multiple broadband, QoS-enabled transport technologies and in which service-related functions are independent from underlying transport-related technologies. It offers unrestricted access by users to different service providers. It supports generalized mobility which will allow consistent and ubiquitous provision of services to users. Además, también se destaca que la NGN se caracteriza, entre otras cosas, por: Decoupling of service provision from network, and provision of open interfaces; Independence of service-related functions from underlying transport technologies (http://www.itu.int/ITU-T/study groups/com13/ngn2004/working_definition.html).

11 A saber: 1) creación de una división mayorista, 2) separación virtual, 3) business separation, 4) business separation con incentivos localizados, 5) business separation wth separate governance arrangements y 6) separación legal. La Directiva 2009/14/CE incorporó la separación funcional como una medida de la que pueden hacer uso las autoridades en caso de persistir problemas de competencia.
} 
la separación funcional, Teppayayon y Bohlin (2010) mencionan como efectos positivos la mayor transparencia de las operaciones del incumbente (lo que hace la discriminación más fácil de detectar), la reducción de los incentivos para dar un trato preferencial a la división minorista del incumbente y la limitación de la discriminación no basada en precios. Como posibles efectos negativos señalan su irreversibilidad, su costosa implementación, la potencial reducción de incentivos a invertir tanto de los incumbentes como de los entrantes y la posible reducción de los incentivos para proveer servicios de calidad.

Por su parte, Gonçalves y Nascimento (2010) proponen un test para evaluar la necesidad de imponer la separación estructural en el caso de las redes de nueva generación. La primera pregunta se refiere al poder de mercado en la provisión de servicios de acceso bajo estas redes ya que en general, se espera que las empresas que comiencen a invertir en esta infraestructura cuenten con un importante poder de mercado (aunque se debe tener en cuenta el papel de las redes de cable en cada mercado). La segunda pregunta se refiere a las potenciales economías de alcance que a su criterio podrían existir entre los diferentes niveles de la estructura vertical. Los autores sostienen que se debe evaluar si medidas alternativas menos drásticas que la separación estructural, como la contabilidad separada o la separación funcional, serían igual de efectivas en el mercado analizado.

Las experiencias de separación funcional o estructural en el sector no son numerosas, y la conveniencia de recurrir a medidas de esta naturaleza podría depender de las circunstancias particulares de cada mercado ${ }^{12}$. Algunos gobiernos han dispuesto condiciones de separación funcional al operador incumbente. Tal es el caso de Reino Unido y Suecia donde la separación funcional surgió como respuesta de las autoridades de regulación ante las escasas muestras de mejoría de la competencia en los mercados de telecomunicaciones ${ }^{13}$. Los resultados de estas medidas no parecen completamente claros. Cadman (2010) encuentra para el caso del Reino Unido que la separación funcional ha permitido incrementar la confianza de los operadores minoristas en que serán tratados en condiciones equivalentes a las del operador minorista de British Telecom, lo que a su vez ha aumentado su disposición a invertir. Sin embargo, no se han percibido efectos más allá de los mercados directamente afectados por la medida. En efecto, en los mercados donde los competidores deben adquirir los insumos directamente de British Telecom y no de Openreach, la empresa incumbente mantiene un comportamiento estratégico. En el caso de Suecia,

\footnotetext{
12 HOWELL et al., (2010) realizan una comparación de las experiencias de separación en el sector eléctrico e intentan encontrar coincidencias entre las características del mercado eléctrico y el de telecomunicaciones con el fin de clarificar los posibles efectos de la imposición de separación estructural en estos últimos.

${ }^{13}$ En ambos casos, los operadores incumbentes (British Telecom y TeliaSonera, respectivamente) optaron voluntariamente por la separación. En el Reino Unido, la separación fue resultado de la negociación entre el regulador y British Telecom, mientras que en Suecia, aún habiendo aceptado la oferta voluntaria de separación funcional de TeliaSonera, el gobierno modificó la Swedish Telecommunications Act en 2008 para incorporar formalmente este tipo de medida en la legislación.
} 
la obligación de provisión de insumos en condiciones equivalentes abarca solamente al bucle local ${ }^{14}$.

Ligado al despliegue de las NGNs, observamos que algunos países frente a las posibilidades que ofrece un nuevo proyecto han decidido promover la separación estructural. En general aquellos gobiernos que están encarando inversiones para la construcción de estas redes, establecen separación estructural entre la operadora de la infraestructura y los proveedores de servicios (tal es el caso de Australia, Nueva Zelanda y Singapur).

\subsection{La intervención del sector público}

Las experiencias de participación pública en el despliegue de redes de nueva generación en el mundo son diversas en cuanto a su motivación, su alcance y su implementación. Entre las razones esgrimidas para llevar a cabo estas inversiones se encuentran criterios de equidad en el acceso a las nuevas tecnologías, la falta de inversión por parte de los operadores privados y la necesidad de invertir en un sector con importantes repercusiones a nivel de producción y consumo. Asimismo, estas redes han representado una alternativa hacia donde dirigir los recursos públicos destinados a la recuperación económica ${ }^{15}$.

En cuanto a la implementación, además de escoger el tipo de estructura industrial que se desea impulsar a través de la regulación como hemos discutido anteriormente, los gobiernos pueden adoptar diferentes esquemas de financiamiento para promover el despliegue de las nuevas redes. Al respecto, la participación pública se ha llevado a cabo de diversas maneras a nivel internacional ${ }^{16}$. En algunas regiones o países los gobiernos han asumido un rol inversor, y el despliegue de la red se ha realizado o se está realizando con fondos 100 por 100 públicos. Algunas experiencias muestran que una vez construida la red la operación de la misma se mantiene en manos públicas, se entrega en concesión a operadores privados o inclusive se vende al cabo de algunos años. En otros casos el gobierno actúa como coinversor y el despliegue de la red se realiza con fondos públicos y privados. En ciertas ocasiones, los gobiernos llevan a cabo un porcentaje mayoritario de la inversión para mantener el control de la red, mientras que en otras tienen una participación minoritaria en la misma.

También han surgido situaciones en las que el gobierno se presenta como proveedor de ayudas o subsidios. Al respecto, los gobiernos pueden decidir subsidiar a

\footnotetext{
${ }^{14}$ La situación frente a los competidores en el contexto del desarrollo de las NGN aún es incierta. Véase TEPPAYAYON y BOHLIN (2010) y CADMAN (2010).

15 Véase CAVE y MARTIN (2010) en relación a los motivos que han llevado a los gobiernos a invertir en el despliegue de redes de nueva generación a escala nacional, a la luz de los casos de Australia, Nueva Zelanda y Singapur. Véase también OECD (2009) y QIANG, C. Z.-W. (2009).

${ }^{16}$ Véase GANUZA et al., (2010) para experiencias de participación pública a nivel internacional bajo diferentes esquemas.
} 
empresas competitivas para que extiendan sus redes en todas las zonas del país o sólo subsidiar el despliegue en zonas no rentables. Al mismo tiempo, los fondos de los subsidios pueden provenir de las arcas generales o se puede plantear un esquema de subsidios cruzados según el cual las zonas rentables financian el despliegue de las zonas no rentables. Finalmente, dado que los costes de despliegue de banda ancha de próxima generación pueden reducirse significativamente si el despliegue se lleva a cabo en el mismo momento que otras obras civiles, los gobiernos pueden aprovechar la ejecución de dichas obras para favorecer la inversión en las nuevas redes de fibra coordinando la actuación con empresas constructoras.

Frente al alto riesgo financiero que conlleva esta inversión, la restricción de los fondos públicos, como así también la importancia de la infraestructura civil en el total de la inversión, las PPPs (public-private partnerships) aparecen como opciones de actuación muy atractivas ${ }^{17}$. Asimismo, las joint ventures entre constructoras acostumbradas a trabajar con largos horizontes temporales y operadoras de telecomunicaciones pueden significar interesantes opciones de negocio ${ }^{18}$.

Al respecto, las posibilidades de colaboración entre el sector público y privado en el contexto del despliegue de las NGNs son más numerosas y complejas que las que se han venido presentando en el sector de telecomunicaciones hasta ahora ${ }^{19}$.

\subsection{El avance de los mercados e industrias online}

El surgimiento y auge de las industrias basadas en Internet están significando importantes desafíos para algunos sectores tradicionales de la economía como la industria literaria, de la música, la publicidad y los medios de comunicación ${ }^{20}$. En

\footnotetext{
${ }^{17}$ Por ejemplo, la Comisión Europea reconoce en las PPPs un instrumento para promover la inversión en sectores de interés estratégico, especialmente en un contexto de crisis económica (COMISIÓN EUROPEA, 2009b).

${ }^{18}$ En este sentido el caso de Holanda es particularmente interesante. En julio de 2008, KPN (la operadora incumbente dueña de la red de cobre) y Reggefiber (una empresa con participación en varios sectores de infraestructura del país y dueña de prácticamente todos los despliegues de fibra óptica que se habían llevado a cabo hasta ese momento) notificaron a la autoridad de competencia holandesa la intención de formar una joint venture para desplegar y operar redes FTTH. Actualmente KPN posee el 41 por 100 de las acciones de Reggefiber y la regulación en relación a la misma incluye: separación funcional y operativa de KPN, provisión de información y obligación de acceso al resto de operadores, precio de acceso a la infraestructura pasiva regulado y no discriminación entre terceros operadores.

19 Véase GÓMEZ-BARROSO y FEIJÓO (2010), que además ofrecen una discusión detallada sobre diferentes modelos de PPPs. NUCCIARELLI et al., (2010) investigan los proyectos con PPPs en Italia y Holanda. Véase FREDEBEUL-KREIN y WERNER KNOBEN (2010) para una explicación de los desafíos a los que se enfrentan las PPPs destinadas al despliegue de NGNs y una revisión de los lineamientos establecidos por la Comisión Europea en relación a las Ayudas Estatales para el despliegue de Banda Ancha (COMISIÓN EUROPEA, 2009a).

${ }^{20} \mathrm{Al}$ respecto, ATHEY et al., (2010) se centran en el hecho de que Internet permite al consumidor moverse fácilmente entre diferentes vendedores. SEAMANS y ZHU (2010) analizan empíricamente el efecto sobre el mercado del periódico local tradicional de la entrada de un sitio web que ofrece servicios de anuncios clasificados. GANUZA y VIECENS (2011a) consideran la posibilidad de desinterme-
} 
este sentido, el sector de las telecomunicaciones no permanece indiferente a los nuevos mercados que aparecen como consecuencia de las posibilidades de acceso a Internet de alta velocidad. En concreto, el sector está siendo invadido por la industria de contenidos online y este hecho se verá potenciado con las posibilidades que ofrecerán las nuevas redes. Un elemento fundamental lo constituye la alternativa con que cuentan los proveedores de contenidos, como eventos deportivos o películas de Hollywood, de ofrecer sus productos a través de portales online. Durante los últimos años algunos operadores de telecomunicaciones han basado sus estrategias de competencia en el acceso a ciertos contenidos premium en exclusividad, o en conseguir suscripciones a sus servicios ofreciendo la posibilidad de acceso a eventos deportivos. El acceso a contenidos via streaming permite la desintermediación, es decir, una vez que el acceso y uso de Internet sea generalizado y dispongamos de altas velocidades (las que posibilitan las NGNs), los dueños y gestores de ciertos contenidos que hasta el momento debían negociar con operadores de telecomunicaciones (o de televisión) para conseguir llegar hasta los consumidores, tendrán la opción de no tener que hacerlo y poder relacionarse con el consumidor directamente a través de una página web ${ }^{21}$. Ganuza y Viecens (2011a) estudian formalmente la interacción entre el desarrollo de la industria de las NGNs y el mercado de contenidos y analizan el impacto que la mencionada posibilidad de desintermediación tendrá sobre los beneficios de la industria de telecomunicaciones. Los autores muestran que la presencia de las NGNs resultará en una reasignación de rentas entre los diferentes agentes de la cadena de valor. En particular, ellos muestran que habrá una transferencia de rentas desde los operadores de redes y servicios a los proveedores de contenidos ${ }^{22}$. La desintermediación por parte de los proveedores de contenidos se traducirá en que las empresas operadoras de servicios de telecomunicaciones perderán una importante fuente de diferenciación e ingresos, por lo que las mismas se verán forzadas a encontrar nuevos recursos para competir. Se deduce que tal vez ha llegado la hora de que las operadoras revisen sus estrategias tradicionales de negocio y refuercen sus departamentos de innovación y desarrollo.

diación que las NGNs dan a los proveedores de contenido, es decir, la posibilidad de llegar a los consumidores evitando a los operadores de telecomunicaciones. Se analiza el impacto de este elemento en los incentivos de los proveedores de contenido para firmar contratos de exclusividad y sobre los beneficios de la industria de telecomunicaciones.

${ }^{21}$ Un ejemplo notable de este hecho surge de la muy recientemente anunciada plataforma de Internet Ultraviolet, www.uvvo.com, creada por los principales estudios cinematográficos de Hollywood (Paramount Pictures, Sony Pictures Entertainment, Twentieth Century Fox, Universal Pictures y Warner Bros entre otros) para ofrecer a los consumidores una gran selección y libertad de compra de películas digitales, programas de televisión y otros entretenimientos via streaming. De manera similar, existen plataformas que permiten acceder a eventos deportivos también a través de streaming.

${ }^{22}$ Una de las principales conclusiones en GANUZA y VIECENS (2011a) es que los proveedores de contenidos elegirán vender sin exclusividad y a través de páginas web aquellos productos que son muy valorados por los consumidores. Esto se traduce en escasos recursos de diferenciación para los operadores de servicios de telecomunicaciones, competencia a la Bertrand y beneficios que tienden a desaparecer para las empresas que prestan servicios de telecomunicaciones. 


\section{Situación en España}

El siguiente mapa muestra que España presenta algunas iniciativas públicas de despliegue de redes de nueva generación, una inversión limitada y difícil de determinar por parte del operador incumbente, Telefónica, y algunas compañías de cable con una tecnología moderna.

GRÁFICO 3

DESPLIEGUES PRIVADOS E INICIATIVAS PÚBLICAS EN ESPAÑA

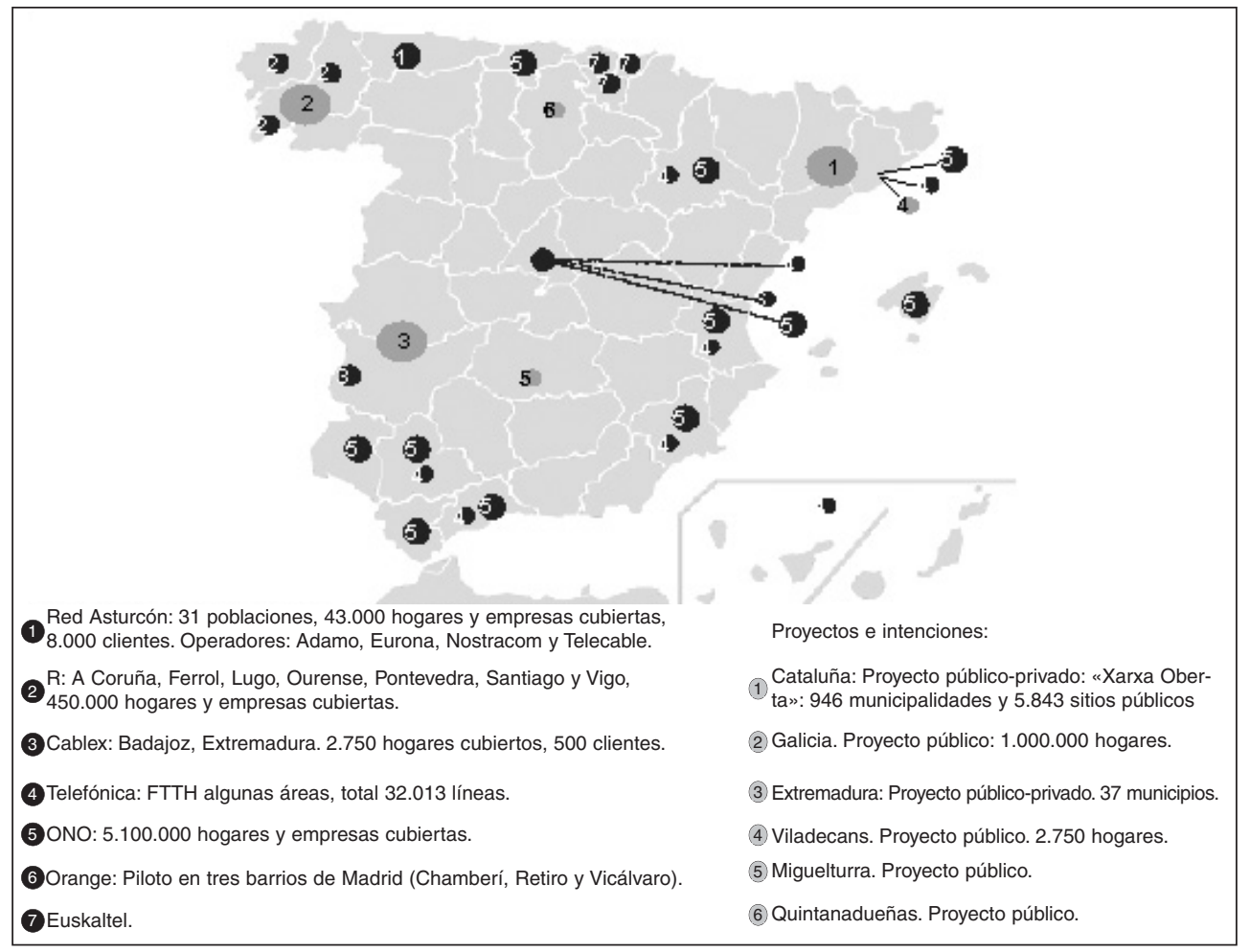

FUENTE: Observatorio Fedea de Redes de Nueva Generación. Enero 2011.

\subsection{Despliegues privados}

El despliegue de Telefónica es escaso y, al menos hasta el momento, difícil de determinar. De acuerdo a información provista en CMT (2010a) Telefónica tiene 32 013 líneas FTTH. Las mismas están distribuidas en algunas áreas localizadas en los puntos (4) del mapa. 
Por otra parte, el cable ofrece cierto potencial para el país. Las compañías de cable en España disponen de una tecnología moderna que puede ser fácilmente actualizada para ser considerada una red de tipo NGAN. En particular, la tecnología DOCSIS 3.0 es ofrecida por ONO en aproximadamente el 70 por 100 de su red, esto es 5,1 millones de hogares y empresas distribuidos en algunas áreas de varias provincias (puntos 5 del Gráfico 3). En este sentido, ONO es en la actualidad la empresa con mayor cobertura para servicios de alta velocidad. Esta tecnología es también provista por $\mathrm{R}$ en algunas ciudades de Galicia, con 450.000 hogares y empresas cubiertas y por Euskaltel en el País Vasco.

Es importante señalar que los puntos en el mapa hacen referencia a la existencia de ciertos despliegues en el área marcada y que los mismos pueden llegar a ser muy limitados. En relación a esto, en Madrid, si bien ONO, Orange y Telefónica están presentes, existen grandes áreas en el centro de la ciudad que no están cubiertas por ninguna red de alta velocidad. Por ejemplo, el caso de Orange corresponde a un despliegue de piloto comercial de FTTH en solo tres barrios (tres de los de mayor renta per cápita en la ciudad). De manera similar, el despliegue en Badajoz llevado a cabo por una empresa local, se ha realizado solamente en dos urbanizaciones residenciales.

\subsection{Iniciativas públicas}

Los tres primeros elementos que destacábamos en la sección 3 se ponen en evidencia en algunos despliegues y proyectos liderados por las Administraciones Públicas españolas.

El caso más paradigmático en este sentido lo constituye la red Asturcom en Asturias, pionera en la provisión de acceso de alta velocidad a la población (la fibra llega hasta los hogares, FTTH). Esta red de propiedad 100 por 100 pública comenzó a desplegarse con una clara intención de llevar actividad económica a la región. La red es abierta, neutra, escalable y estructuralmente separada del mercado de servicios minoristas. Los clientes de la red son los operadores de servicios (en la actualidad hay 4 empresas) que se conectan a un punto de la red para proveer los servicios finales a sus respectivos suscriptores. Los operadores compiten por las suscripciones y pagan una tarifa de acceso por cada suscripción. La red llega a ciudades con más de 1.000 habitantes pero no está presente en las grandes ciudades. La financiación de la inversión proviene de fondos públicos (en particular de «fondos mineros»).

Algunos municipios, tales como Viladecans, Quintanadueñas y Miguelturra, están aprovechando los fondos del Plan E (a través de los planes Avanza ${ }^{23}$ ) para pro-

${ }^{23}$ Los planes Avanza constituyen dinero que las Comunidades Autónomas y Municipios pueden solicitar para promover el desarrollo de la Sociedad de la Información y del Conocimiento. Corresponden al Ministerio de Industria, Turismo y Comercio, y son canalizados a través de la Secretaría de Estado de Telecomunicaciones y para la Sociedad de la Información. Hasta el momento se han destinado aproximadamente 6.500 millones de euros, de los cuales unos 538 millones de euros se han destinado a programas de extensión de Banda Ancha (www.planavanza.es). 
poner proyectos para el acceso a la fibra. Todos ellos son poblaciones muy pequeñas cuyos gobiernos argumentan que la inversión privada no llegará a sus regiones.

Hay otros dos proyectos en marcha que, de hacerse realidad, podrían adquirir una gran relevancia en el país: el «Plan de Banda Larga 2010-2013» en Galicia y el proyecto de Extremadura liderado por «Telecable-SOFIEX». El plan de Galicia pretende que un millón de habitantes de áreas que no son rentables para el sector privado tengan acceso a Internet con velocidades de $100 \mathrm{Mbps}$. Al respecto, en noviembre de 2010 la CMT autorizó un proyecto que consiste en el otorgamiento de una subvención por un importe máximo de 28 millones de euros a un operador privado, $\mathrm{R} \mathrm{Cable}^{24}$ (seleccionado mediante un procedimiento competitivo) para la construcción de una red de acceso de nueva generación ${ }^{25}$. De los 329 núcleos del territorio gallego con más de 500 habitantes, el adjudicatario deberá establecer la red y prestar los servicios en al menos 86. Los despliegues de red propuestos deberán proporcionar servicios de acceso de nueva generación, por lo menos, a 355.000 habitantes. En los 86 núcleos el beneficiario deberá ofrecer: acceso a un paquete de servicios minoristas al menos en el 50 por 100 de los inmuebles y acceso a los servicios minoristas en todos los edificios de la Administración ${ }^{26}$. R Cable además tendrá la obligación de proveer servicio mayorista de manera neutra y abierta a terceros operadores por un precio orientado a costes ${ }^{27}$.

En Extremadura se ha anunciado la intención de llevar adelante un proyecto liderado por Telecable (empresa privada) para llevar la fibra a 37 ciudades en los próximos 10 años y ofrecer a sus habitantes conexiones con velocidades de $100 \mathrm{Mbps}$ y 1 Gbps. Éste es un proyecto de participación pública y privada, que incluye a Telecable con una participación del 51 por 100 y a SOFIEX (propiedad del gobierno de Extremadura en un 96,99 por 100) con el 49 por 100 restante $^{28}$.

Finalmente, la «Xarxa Oberta» es un proyecto público-privado para implementar las NGNs en Cataluña y que pretende llegar con la fibra óptica a todos los municipios (946) y a las 5.843 sedes públicas de la Comunidad. Se incluye toda agencia

\footnotetext{
${ }^{24}$ Estos 28 millones son cofinanciados al 80 por 100 por el Fondo Europeo de Desarrollo Regional (FEDER). El operador privado deberá aportar además 42 millones de euros, por lo que la inversión total será de 70 millones de euros.

25 RE-2010-11-18-2-1 de la CMT: Resolución por la que se aprueba un informe a la Xunta de Galicia sobre un proyecto de ayudas para el despliegue de redes de acceso de nueva generación en el ámbito de la Comunidad Autónoma de Galicia (MTZ 2010-896). En esta resolución se muestra que solo las 7 ciudades más grandes y que representan el 36 por 100 de la población de la Comunidad estarían dentro de la clasificación de «Zona negra/gris», es decir, son núcleos de población con presencia o previsión de despliegue de red de acceso de banda ancha ultrarrápida de más de un operador en un futuro próximo. De acuerdo con las Directrices comunitarias (COMISIÓN EUROPEA, 2009a), en estas zonas los gobiernos no pueden otorgar ayudas públicas para promover las NGNs.

${ }^{26} \mathrm{Se}$ exige $100 \mathrm{Mbit} / \mathrm{s}$ descendentes y $5 \mathrm{Mbit} / \mathrm{s}$ ascendentes.

27 La convocatoria incluye una cláusula de reversión con el objeto de evitar que no se compense en exceso a los beneficiarios de la ayuda por una demanda de banda ancha superior a los niveles previstos y la posibilidad de utilizar infraestructuras ya desplegadas. El proyecto ya ha sido notificado por la Xunta de Galicia a la Comisión Europea autorización en el marco de las Ayudas de Estado.

28 Para más detalles, véase http://www.sofiex.es/.
} 
y departamento gubernamental, instituciones de salud, educación e investigación, museos, bibliotecas, servicios de policía, etc. Además, la red será neutral, abierta, estructuralmente separada y el operador de la red deberá proveer acceso mayorista a todos los operadores que deseen prestar servicios en el mercado minorista. El proyecto ha sido aprobado en agosto 2010 por la Comisión Europea ${ }^{29}$, en particular, la Comisión concluyó que el proyecto «Xarxa Oberta» cumple con los criterios de compatibilidad establecidos en las Directrices Comunitarias de Banda Ancha (Comisión Europea, 2009a) ${ }^{30}$.

\subsection{España en perspectiva internacional}

En la sección anterior hemos revisado las iniciativas que existen en España para otorgar a los consumidores posibilidades de acceso a Internet de alta velocidad. Hemos observado que las mismas son hasta la fecha de alcance muy limitado. En esta sección llevamos a cabo un análisis comparativo de la situación en España en relación al resto del mundo.

El primer hecho que resalta es la tradición que tiene el país de baja penetración de banda ancha y de precios entre los más altos de Europa. En particular, las estadísticas presentadas recientemente por la OCDE representan una evidencia contundente de que la situación de España es preocupante (OCDE, 2010). En términos de penetración de banda ancha, España ocupa el puesto número 20 dentro de un grupo de 30 países como se observa en el Gráfico $4^{31}$. Además, mientras que la tasa de penetración promedio de los países de la OCDE es 24.2 la correspondiente a España es 22.2 .

\footnotetext{
${ }^{29}$ Decisión de la Comisión en el Caso N407/2009, Fibra Óptica en Cataluña 12/08/10.

30 Para un análisis detallado del proyecto Xarxa Oberta (licitación, caso en la Comisión Europea, etc.), véase GANUZA y VIECENS (2011b).

$31 \mathrm{Al}$ mismo tiempo, con el paso de los años España ha ido cayendo en relación al resto de países. Por ejemplo, en el 2002 ocupaba el lugar 11 y en el 2007 el 19 (KOUTROUMPIS, 2009).
} 


\section{GRÁFICO 4}

TASA DE PENETRACIÓN PAÍSES DE LA OCDE (TOTAL DE LÍNEAS FIJAS POR CADA 100 HABITANTES)

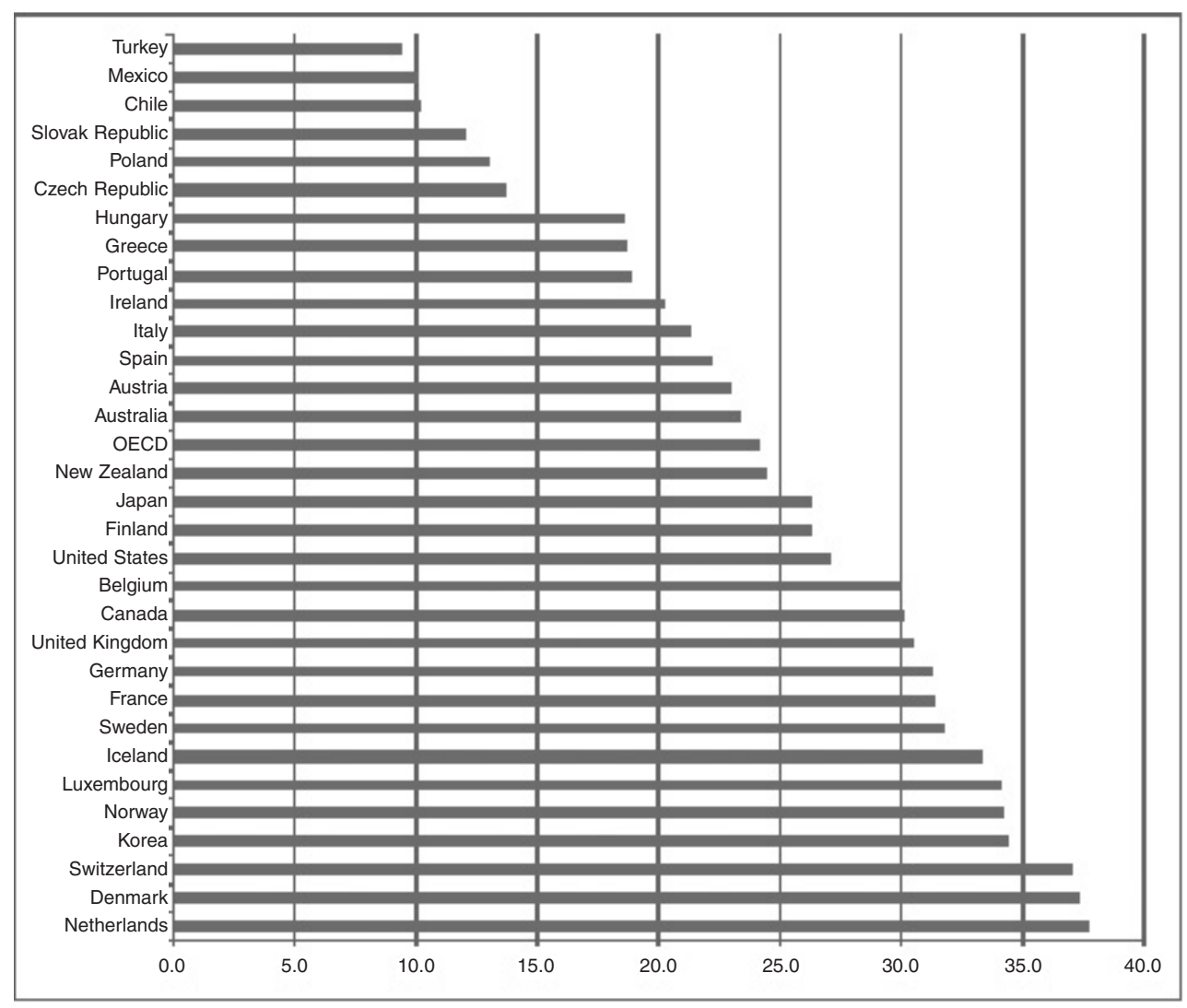

FUENTE: OCDE (2010). Junio de 2010.

En relación a las Redes de Nueva Generación, España no está bien posicionada en los rankings sobre acceso, y en particular, no aparece entre los primeros 20 países con mayor penetración de la fibra hasta el hogar elaborado por el FTTH Council Europe ${ }^{32}$. De manera similar, si se comparan las cifras de penetración de servicios basados en redes de nueva generación en España con el resto de países de la OCDE, teniendo en cuenta tanto las tecnologías FTTx como las de cable, la brecha es evidente. Debido a la escasa cobertura de estas redes y a su reciente despliegue, la mayor parte de los accesos a Internet de banda ancha en España corresponden a accesos ADSL. La siguiente figura muestra los porcentajes de suscripciones de banda ancha con tecnología de fibra/LAN y con cable. En el ranking de fibra España ocupa el puesto 23, y en el correspondiente al cable ocupa el 19.

\footnotetext{
${ }^{32}$ http://www.ftthcouncil.eu.
} 


\section{GRÁFICO 5 \\ SUSCRIPCIONES A FIBRA/LAN Y CABLE CADA 100 HABITANTES (PAÍSES DE LA OCDE)}

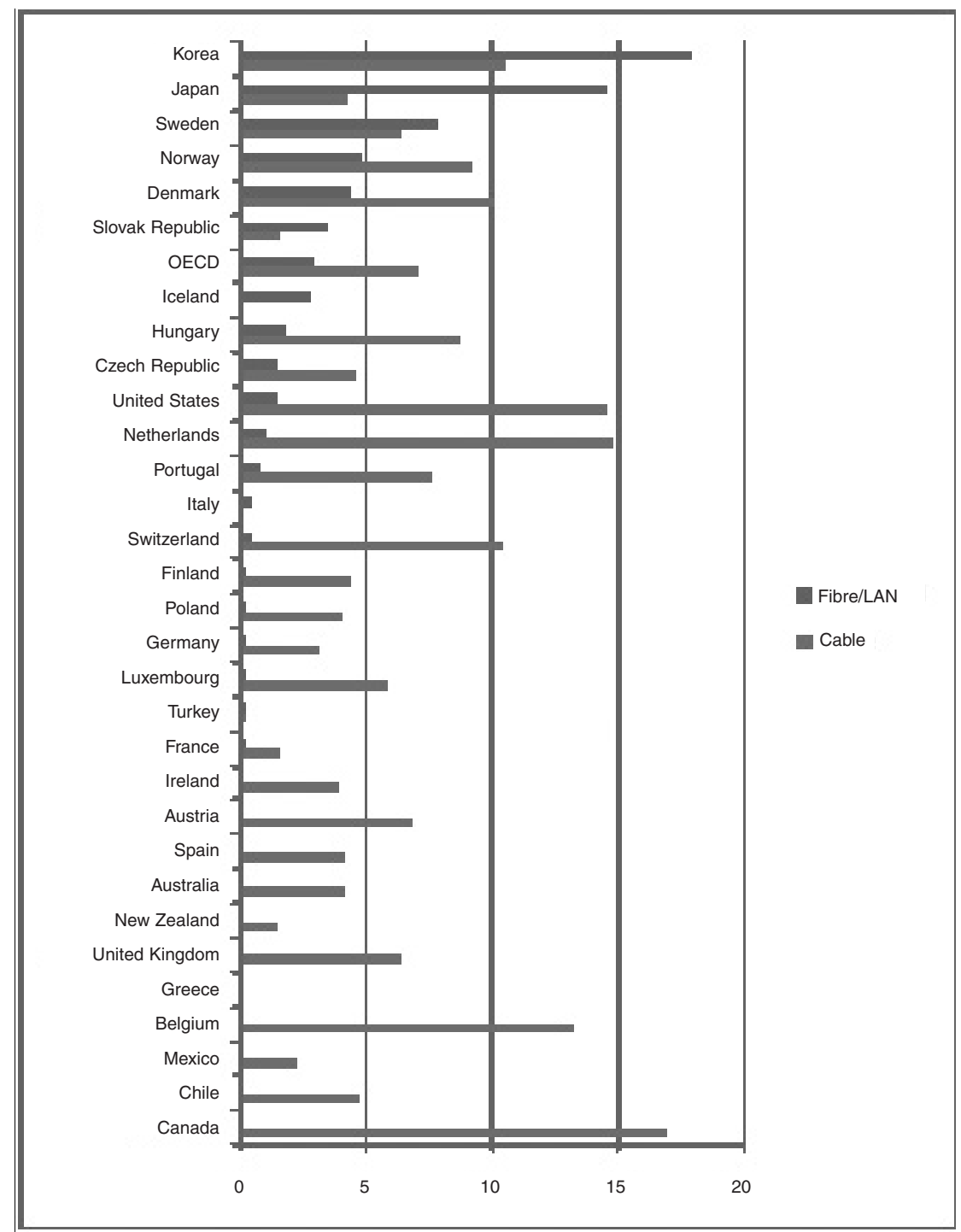

FUENTE: OCDE (2010). Junio de 2010.

En relación a los precios que pagan los consumidores, el último informe comparativo sobre los precios de banda ancha en la Unión Europea elaborado por la Autoridad Nacional Regulatoria de España (CMT, 2010b) muestra que los servicios de banda ancha y voz con velocidad media (de 2 Mbps a 10 Mbps) son un 10,8 por 100 más caros en España que la media de las mejores ofertas en Europa. Por otra parte, cuan- 
do el estudio se limita a comparar solo las ofertas de los operadores tradicionales, se muestra que la mejor oferta del operador histórico español (Telefónica) es un 71,7 por 100 más cara que la media de las mejores ofertas de otros operadores en Europa.

\section{Conclusiones}

En este documento hemos mencionado cuatro elementos que vienen ligados al despliegue de las redes de nueva generación y que implican importantes novedades para el sector de las telecomunicaciones como así también serios desafíos para los agentes tradicionales que operan en el mismo. Al respecto, hemos resaltado la reaparición de la intervención del sector público lo que pone en evidencia un alejamiento del consenso de las últimas dos décadas en cuestiones de política de liberalización y privatización (Given, 2010).

Luego, hemos señalado que estas redes están generando un nuevo debate sobre la conveniencia de contar con operadores de redes separados del nivel de servicios al consumidor final y hemos destacado el surgimiento de redes de fibra descentralizadas y «pequeñas», en contraste con las grandes redes centralizadas de cobre. Finalmente, hemos destacado el impacto que la industria online representa para el sector de las telecomunicaciones y cómo ese impacto se verá reforzado con la presencia de las nuevas redes y mayores velocidades de acceso.

Un aspecto sobre el que no hemos hablado pero que también merece atención es el impacto de la convergencia tecnológica en la organización industrial de las telecomunicaciones. Este fenómeno tecnológico, que potencialmente se verá reforzado con la presencia de las nuevas redes, repercute inevitablemente en la estructura y modelo de competencia del sector como así también en sus necesidades regulatorias. Este elemento contribuye así a reforzar la hipótesis de que las redes de acceso de nueva generación llegan al sector para revolucionarlo por completo.

La segunda parte del artículo se ha centrado en describir y evaluar la situación de las NGNs en España con respecto a los demás de países de la OCDE. La conclusión global es que el despliegue de las NGANs es muy modesto. Esta situación se explica en parte por la falta de incentivos de Telefónica a invertir. Las empresas de telecomunicaciones tradicionales, como Telefónica, son en general muy prudentes con respecto a las NGANs, dado que las NGANs tienen muchos riesgos (los beneficios de la cadena de valor tienden a desplazarse hacia los proveedores de contenidos), y por ende la rentabilidad no está asegurada ${ }^{33}$. Por otra parte, el despliegue de las NGANs reduciría drásticamente el valor de sus activos actuales (ADSL, telefonía fija, etcétera), algo que puede ser un factor importante para Telefónica dada su alta cuota de mercado. Por ejemplo, las ofertas combinadas en España de telefonía, TV y ADSL, tienen el mismo precio (o inclusive más alto) que el de las ofertas europeas

\footnotetext{
33 Véase también HUIGEN y CAVE (2008).
} 
de televisión y TV con Internet a $100 \mathrm{Mbps}$ (es decir con una red de acceso de nueva generación $)^{34}$. ¿Qué incentivo tiene una empresa a invertir 600 Euros por consumidor si finalmente podrá cobrarle lo mismo? Por último, Telefónica no está sujeta a una fuerte presión competitiva. Ésta es una empresa muy grande, con respaldo financiero y buenas capacidades tecnológicas por lo que sería capaz de reaccionar muy rápido ante cualquier amenaza de inversión en el mercado interno por parte de algún competidor. Por lo tanto, Telefónica parece seguir una estrategia racional de «esperar y ver» en relación a las NGANs ${ }^{35}$. Hasta la actualidad, el mayor esfuerzo de despliegue de NGANs se ha llevado a cabo por la compañía de cable ONO, cuyas cuotas de mercado son del 14,8 por 100 en líneas de banda ancha y de 9 por 100 en telefonía fija (CMT, 2010a). La red de ONO puede ser actualizada a DOCSIS $3.0 \mathrm{sin}$ grandes esfuerzos económicos. Sin embargo, las limitaciones financieras de la empresa acotan su capacidad para invertir. Ahora, ONO está más concentrada en la actualización de su red existente que en la ampliación de la misma.

Por lo tanto, la conclusión de la evaluación de la situación actual es que España está retrasada en el despliegue de las NGANs. Además, no existe ningún indicio claro que haga pensar que esta situación vaya a revertirse a corto/medio plazo. Por los argumentos expuestos con anterioridad, en el futuro próximo no es probable que la inversión privada desempeñe un papel importante en el despliegue de las NGANs en España. Además, a pesar de las iniciativas de algunos gobiernos autonómicos, no parece que el esfuerzo del sector público en el despliegue de las NGANs vaya a ser muy relevante. España todavía no ha puesto en marcha un plan nacional de banda ancha. La situación actual de España con un fuerte déficit fiscal implica que el país, en caso de pretender hacerlo, no sería capaz de invertir en redes de acceso de nueva generación al mismo nivel que lo están haciendo otros países. Por ejemplo, Australia, un país con el mismo nivel de penetración que España, con una empresa establecida con una gran cuota de mercado, precios altos y porcentajes de acceso por tecnología muy similares a España, está llevando adelante un proyecto de despliegue a escala nacional impulsado por el gobierno (Given, 2010). El futuro próximo nos dirá si la divergencia en las políticas adoptadas por Australia y España se traducirá en que estos dos países, que ahora presentan un diagnóstico similar, muestren importantes diferencias en el nivel de competencia y desarrollo del sector.

\footnotetext{
34 En Francia, Orange ofrece un paquete de este tipo que incluye 100 Mbps de velocidad de bajada por 34,90 euros más IVA. Mientras tanto, Movistar (Telefónica) presenta una oferta con 10 Mbps por 54,80 euros más IVA (páginas web de la empresas operadoras en noviembre de 2010).

35 La regulación y/o la incertidumbre regulatoria podrían contribuir también a explicar la falta de incentivos a invertir por parte de los operadores privados. En este sentido, la literatura no es concluyente sobre el efecto de la regulación en los incentivos a invertir (véase CAMBINI y JIANG (2009) para una revisión de esta cuestión). En España, la CMT ha impuesto a Telefónica la obligación de prestar un servicio de acceso indirecto solo hasta los $30 \mathrm{Mbps}$ a sus competidores (http://www.cmt.es/es/ documentacion_de_referencia/mercados_comunicaciones_electronicas/anexos/Resolucion_mercados_4_y_5.pdf). Sin embargo, a la luz de la última recomendación de la Comisión Europea (Comisión Europea, 2010b), la CMT podría verse obligada a ampliar las obligaciones de acceso para velocidades mayores.
} 


\section{Referencias}

[1] ATHEY, S.; CALVANO, E. y GANS, J. S. (2010): Will the Internet Destroy the News Media? or Can Online Advertising Markets save the Media? Mimeo.

[2] CADMAN, R. (2010): Means not ends: Deterring discrimination through equivalence and functional separation, Telecommunications Policy 34, 366-374.

[3] CAVE, M. (2006): Six degrees of separation: operational separation as a remedy in European telecommunications regulation, Communications and Strategies, 64, 89-103.

[4] CAVE, M. y MARTIN, I. (2010): Motives and means for public investment in nationwide next generation networks, Telecommunications Policy 34, 505-512.

[5] CAMBINI, C. y JIANG, Y. (2009): Broadband investment and regulation: A literature review. Telecommunications Policy, 33, 559-574.

[6] CMT (2010a): Estadísticas del sector, III trimestre-2010.

[7] CMT (2010b): Comparativa internacional de ofertas comerciales de banda ancha en la Unión Europea.

[8] COMISIÓN EUROPEA (2009a): Directrices comunitarias para la aplicación de las normas sobre ayudas estatales al despliegue rápido de redes de banda ancha. Diario Oficial de la Unión Europea, 2009/C 235/04.

[9] COMISIÓN EUROPEA (2009b): Movilizar las inversiones públicas y privadas con vistas a la recuperación y el cambio estructural a largo plazo: desarrollo de la colaboración público-privada $(C P P)$.

[10] DOYLE, C. (2008): Structural separation and investment in the National Broadband Network Environment, a Report for Optus.

[11] FORNEFEL, M.; DELAUBAY, G. y ELIXMANN, D. (2008): The Impact of Broadband on Growth and Productivity, a study on behalf of the European Commission.

[12] FREDEBEUL-KREIN, M. y KNOBEN, W. (2010): Long term risk sharing contracts as an approach to establish public-private partnerships for investment into next generation access networks, Telecommunications Policy 34, 528-539.

[13] GANUZA, J. J.; PERCA, K. y VIECENS, M. F. (2010): Las Redes de Nueva Generación en España. Situación actual y retos para el futuro, FEDEA Estudios Económicos 02-2010.

[14] GANUZA, J. J. y VIECENS, M. F. (2011a): Exclusive Content and Next Generation Networks, mimeo FEDEA.

[15] GANUZA, J. J. y VIECENS, M. F. (2011b): Deployment of High-speed Broadband Infrastructures during the Economic Crisis. The case of Xarxa Oberta. De próxima publicación en Telecommunication Policy.

[16] GAPTEL (2008): Oportunidades y Desafíos de la Banda Ancha.

[17] GIVEN, J. (2010): Take your partners: Public private interplay in Australian and New Zealand plans for next generation broadband, Telecommunications Policy 34, 540-549.

[18] GÓMEZ-BARROSO, J. L. y FEIJOO, C. (2010): A conceptual framework for publi-private interplay in the telecommunications sector, Telecommunications Policy 34,487-495.

[19] GONÇALVES, R. y NASCIMENTO, A. (2010): The momentum for network separation: A guide for regulators, Telecommunications Policy 34, 355-365.

[20] HOLT, L. y JAMISON, M. (2009): Broadband and contributions to economic growth: Lessons from the US experience, Telecommunications Policy, 33, 575-581. 
[21] HOWELL, B.; MEADE, R. y O'CONNOR, S. (2010): Structural separation versus vertical integration: Lessons for telecommunications from electricity reforms, Telecommunications Policy 34, 392-403.

[22] HUIGEN, J. y CAVE, M. (2008): Regulation and the promotion of investment in next generation networks-A European dilemma. Telecommunications Policy, 32, 713-721.

[23] KATZ, R. y SUTER, S. (2009): Estimating the economic impact of the US broadband stimulus plan, Columbia Institute for Tele-Information. Working Paper.

[24] KATZ, R.; VATERLAUS, S.; ZENHÄUSERN, P.; SUTER, S. y MAHLER, P. (2009): The Impact of Broadband on Jobs and the German Economy, Columbia Institute for tele-Information, Working Paper.

[25] KOUTROUMPIS, P. (2009): The economic impact of broadband on growth: a simultaneous approach, Telecommunications Policy, 33, 471-485.

[26] MITCHELL, S. y PEZZULLO, L. (2010): Financial and externality impacts of highspeed broadband for telehealth. Informe Access Economics encargado por el Gobierno de Australia (Department of Broadband, Communications and the Digital Economy).

[27] NUCCIARELLI, A.; SADOWSKI, B. M. y ACHARD, P. (2010): Emerging models of public-private interplay for European broadband access: evidence from the Netherlands and Italy, Telecommunications Policy, 34, 513-527.

[28] OCDE (2009): The role of communication infrastructure investment in economic recovery.

[29] OCDE (2010): Indicators of broadband coverage, OECD, París.

[30] SEAMANS, R. y ZHU, F. (2010): Technology Shocks in Multi-Sided Markets: The Impact of Craigslist on Local Newspapers, NET Institute Working Paper No. 10-11.

[31] SIMES, R.; HUTLEY, N.; HAVYATT, S. y McKIBBIN, R. (2010): Australian Business Expectations for the National Broadband Network. Informe Access Economics.

[32] TEPPAYAYON, O. y BOHLIN, E. (2010): Functional separation in Swedish broadband market: Next step of improving competition, Telecommunications Policy 34, 375-383.

[33] QIANG, C. Z.-W. (2009): Broadband Infrstructure in Stimulus Packages: Relevant for Developing Countries, World Bank. 


\section{CUADERNOS ECONÓMICOS DE INFORMACIÓN COMERCIAL ESPAÑOLA (CICE)}

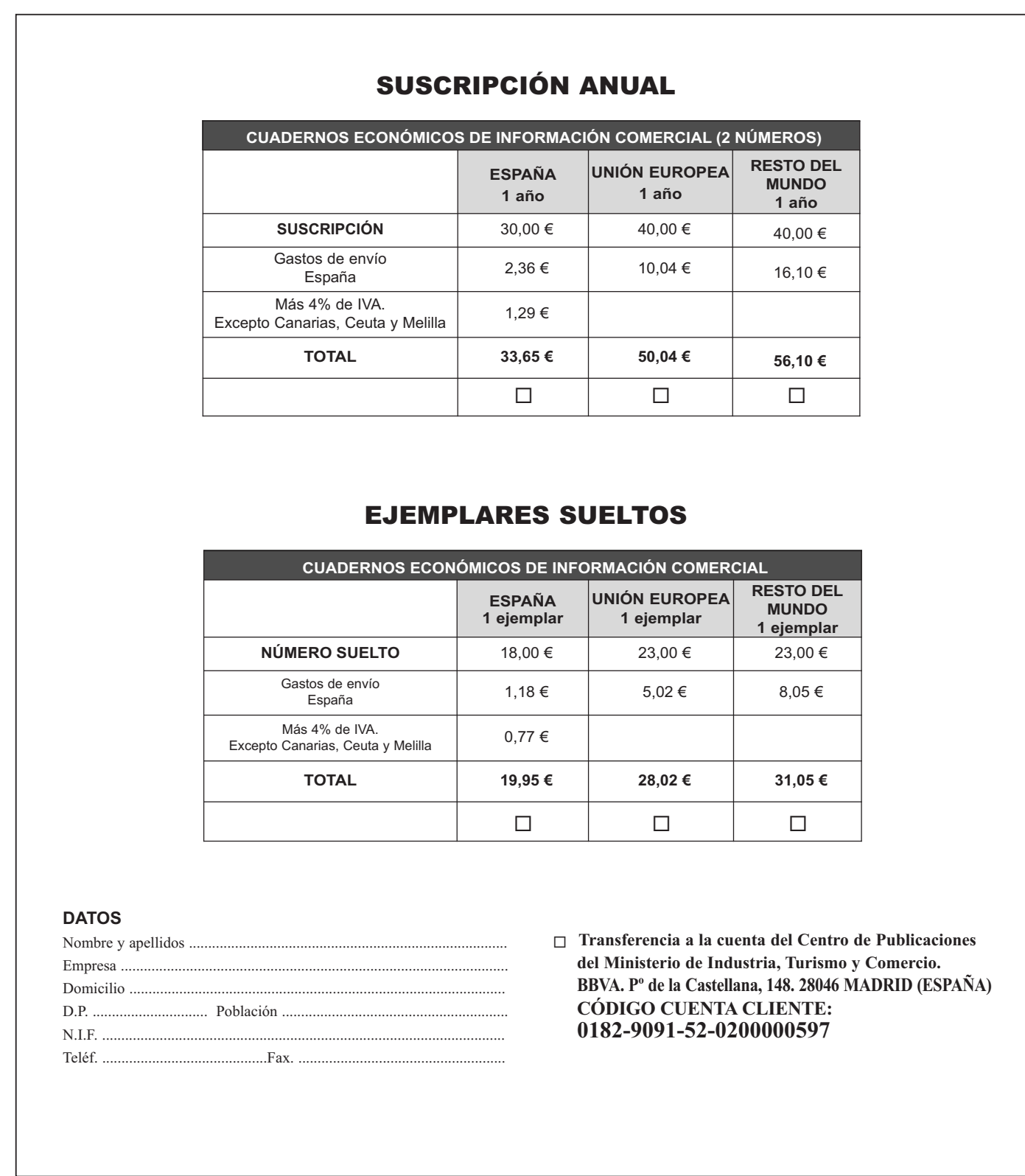

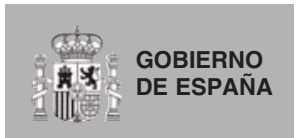

$\begin{array}{ll} & \text { SUBSECRETARÍA } \\ \text { MINISTERIO } & \text { SECRETARÍA GENERAL TÉCNICA } \\ \text { DE INDUSTRIA, TURISMO } & \text { SUBDIRECIÓN GENERAL } \\ \text { Y COMERCIO } & \text { DE DEARROLLO NORMATIVO, } \\ \text { INFORMES Y PUBLICACIONES } \\ \text { CENTRO DE PUBLICACIONES }\end{array}$

Información y venta directa:

Calle Panamá, 1. Vestíbulo. 28071 Madrid. Teléfono 913497605 / 3494968

Suscripciones y ventas por correspondencia:

Calle Panamá, 1. Planta 0. 28071 Madrid. Teléfono 9134951 29. Fax: 913494485

Suscripciones a través de la página web del Ministerio de Industria, Turismo y Comercio:

http://www.revistasice.com/RevistasICE/Suscripciones/pagFormulario.htm 